\title{
Corrigendum
}

\section{Rho modulates hepatic sinusoidal endothelial fenestrae via regulation of the actin cytoskeleton in rat endothelial cells}

Hiroaki Yokomori, Kazunori Yoshimura, Shinsuke Funakoshi, Toshihiro Nagai, Kayo Fujimaki, Masahiko Nomura, Hiromasa Ishii and Masaya Oda

Laboratory Investigation (2004) 84, 1079. doi:10.1038/labinvest.3700138

Correction to: Laboratory Investigation (2004) 84, 857-864. doi:10.1038/labinvest.1300353

Following publication of the above paper, the author has identified errors with the following two sentences. The corrections are:

Page 859, third paragraph-The samples $(30 \mu \mathrm{g}$ protein/well) were separated by sodium dodecyl sulfate-polyacrylamide gel electrophoresis (SDS-
PAGE) under nonreducing condition on $75 \%$ acrylamide gel, and electrophoretically transferred to a PVDF (Millipore, Bedford, MA, USA).

Page 862, first paragraph-In the Western blot, phosphorylated myosin light-chain protein expression was high in LPA-treated SEC, but very low in C3-transferase-treated cells (Figure 4), while Rho A expression levels were similar in the three groups. 